\title{
Asymptotics of the information entropy of the Airy function
}

\author{
P Sánchez-Moreno † T, R J Yáñez $\$$ §and J S Dehesa †§ \\ † Departamento de Física Moderna, Universidad de Granada, Granada, Spain \\ † Departamento de Matemática Aplicada, Universidad de Granada, Granada, Spain \\ $\S$ Instituto "Carlos I" de Física Teórica y Computacional, Universidad de Granada, \\ Granada, Spain.
}

\begin{abstract}
The Boltzmann-Shannon information entropy of the linear potential wavefunctions is known to be controlled by the information entropy of the Airy function $\operatorname{Ai}(x)$. Here, the entropy asymptotics is analyzed so that the leading term (previously calculated in the WKB approximation) as well as the following term (already conjectured) are derived by using only the specific properties of the Airy function.

E-mail: pablos@ugr.es
\end{abstract}




\section{Introduction.}

The Boltzmann-Shannon information entropy of an individual microstate of a onedimensional physical system is defined by

$$
S[\Psi]:=-\int|\Psi(x)|^{2} \log |\Psi(x)|^{2} d x,
$$

where $\Psi(x)$ is the quantum-mechanical wavefunction of the state $[1,2]$. The exact computation of this quantity is a formidable, practically impossible task. This is because either the Schrödinger equation of the system cannot be exactly solved or, when it is, the wavefunction is a known special function whose information entropy cannot be analytically evaluated and its numerical computation is highly unstable. Most efforts have been focussed on classical hypergeometric functions of the type $\Psi(x)=\sqrt{\omega(x)} y_{n}(x)$, where $\left\{y_{n}(x) ; n=0,1, \ldots\right\}$ denotes a set of polynomials orthogonal with respect to the weight function $\omega(x)$. In this case, the computation of the entropy (1) boils down to evaluating the so-called information entropy of the orthogonal polynomials $y_{n}(x)$, given by

$$
S\left[y_{n}\right]:=-\int y_{n}^{2}(x)\left[\log y_{n}^{2}(x)\right] \omega(x) d x .
$$

Briefly, up to now, this mathematical quantity has been exactly calculated only for Chebyshev polynomials, although numerous results for other classical orthogonal polynomials (Hermite, Laguerre, Jacobi) have been found, mainly from an asymptotical point of view. See Ref. [3] for a recent summary of the main achievements. The numerical computation of this entropic integral on finite intervals is most conveniently done by the effective method of Buyarov et al. [4].

Recently, the evaluation of the physical entropy (1) has been attacked for wavefunctions controlled by special functions other than orthogonal polynomials. This occurs for systems such as the circular membrane [5], the Toda-like potential [6] and confining power-type potential [7]. For these cases the entropy has been evaluated in the asymptotic region, that is for highly energetic quantum states, either by use of only the properties of the involved special functions or by means of the semiclassical or WKB approximation. In doing so, we have calculated the asymptotics of the information entropies of the Bessel functions [5] and the Mcdonald functions [6].

In this paper we shall consider the computation of the Boltzmann-Shannon entropy for a linear potential, i.e. for the quantum-mechanical problem of a particle under the influence of a constant (nonvanishing) force. This potential for vanishing orbital quantum number and the harmonic oscillator potential are the only two power-type confinement potentials $[8,9]$, so much used with quark models and more particularly the charmonium model [10] of the $J / \psi$ particle $[11,12]$, which are exactly solvable in terms of known special functions. The linear potential has the same importance in particle physics as the Coulomb potential in atomic physics and the harmonic potential in solid state physics. In Section 2 it is pointed out that the linear potential wavefunctions for vanishing orbital quantum number are controlled by the convergent 
solutions of Airy's equation, i.e. by the Airy function $\operatorname{Ai}(z)$. So, the calculation of the physical entropy of the highly energetic states of a particle in the linear potential boils down to the asymptotics of the information entropy of the Airy function. The relation between these physical and mathematical quantities was explicitly given by J. Sánchez-Ruiz [13]. This author not only determined the leading terms of the entropy by use of the WKB approximation, but also conjectured the following term in the form $K^{\prime} n^{\prime-1 / 3}$, with $K^{\prime} \simeq 0.709$ if we consider $n^{\prime}=1,2,3 \ldots$ running only over odd states, or $K \simeq 2^{1 / 3} K^{\prime}=0.8933$ if we consider $n=1,2,3 \ldots$ running over odd and even states. Notice that $K^{\prime} n^{-1 / 3}=K n^{-1 / 3}=K\left(2 n^{\prime}+1\right)^{-1 / 3} \simeq K 2^{-1 / 3} n^{-1 / 3}$.

The main purpose of this paper is the analytical derivation of these two terms by means of the specific properties of the Airy functions, although the value of $K$ is numerically calculated. The structure of this paper follows so that in Section 3, the asymptotics of the entropy of the Airy function $S_{n}^{\mathrm{Ai}}$ is investigated in detail by use of its specific properties. Then, in Section 4 , the constant $K$ is numerically evaluated. Finally, a brief summary and some open problems are pointed out.

\section{Linear potential wavefunctions and the Airy function: information entropies.}

The three-dimensional motion of a particle under a linear potential for the case of vanishing orbital quantum number reduces to the one-dimensional problem of the potential $V(x)=F|x|$, where $F$ denotes the field strength $[14,15]$. The wavefunctions of the quantum-mechanical states of the particle in such a potential are the solutions of the associated Schrödinger equation,

$$
-\frac{1}{2} \frac{d^{2} \Psi(x)}{d x^{2}}+F|x| \Psi(x)=E \Psi(x)
$$

where units $\hbar=m=1$ have been used. Various authors (see e.g. [14,15]) have shown that the eigenfunctions can be expressed in terms of the Airy function as

$$
\Psi_{n}(x)=N_{n} \operatorname{Ai}\left(\alpha|\mathrm{x}|+\beta_{\mathrm{n}}\right),
$$

where the normalization contant $N_{n}$ and the parameters $\beta_{n}$ and $\alpha$ are given by

$$
\begin{aligned}
& N_{n}= \begin{cases}\sqrt{\frac{\alpha}{2}} \frac{1}{\sqrt{-a_{n / 2+1}^{\prime}} \operatorname{Ai}\left(\mathrm{a}_{\mathrm{n} / 2+1}^{\prime}\right)} & \text { evenn } \\
\operatorname{sign}(\mathrm{x}) \sqrt{\frac{\alpha}{2}} \frac{1}{\mathrm{Ai}^{\prime}\left(\mathrm{a}_{(\mathrm{n}+1) / 2}\right)} & \text { oddn }\end{cases} \\
& \beta_{n}=\left\{\begin{array}{ll}
a_{n / 2+1}^{\prime} & \text { evenn } \\
a_{(n+1) / 2} & \text { oddn }
\end{array},\right. \\
& \alpha=(2 F)^{1 / 3},
\end{aligned}
$$

where $a_{s}$ and $a_{s}^{\prime}$ are the zeros of the Airy function $\mathrm{Ai}(\mathrm{x})$ and its derivative $\mathrm{Ai}^{\prime}(\mathrm{x})$ respectively. 
The associated energy eigenvalues are

$$
E_{n}=-\frac{F}{\alpha} \beta_{n}
$$

The spreading of the linear potential wavefunctions, $\Psi_{n}(x, t)=\Psi_{n}(x) \exp \left(-\frac{i}{\hbar} E_{n} t\right)$, (3)-(2), is best described [1] by the Boltzmann-Shannon information entropy (1), which gives the spatial distribution of the associated quantum-mechanical Born density $\left|\Psi_{n}(x, t)\right|^{2}=\left|\Psi_{n}(x)\right|^{2}$. Moreover, it gives the uncertainty of the position of the particle. Taking into account Eq. (2), this entropy is expressed as

$$
S\left[\Psi_{n}\right]=-\int_{-\infty}^{\infty}\left|\Psi_{n}(x)\right|^{2} \log \left|\Psi_{n}(x)\right|^{2} d x=-\log N_{n}^{2}+2 \frac{N_{n}^{2}}{\alpha} S_{n}^{\mathrm{Ai}},
$$

where $S_{n}^{\mathrm{Ai}}$ is the information entropy of the Airy function on the interval $\left[\beta_{n}, \infty\right)$ :

$$
S_{n}^{\mathrm{Ai}}=-\int_{\beta_{n}}^{\infty} \mathrm{Ai}^{2}(\mathrm{x}) \log \mathrm{Ai}^{2}(\mathrm{x}) \mathrm{dx} .
$$

\section{Entropy asymptotics.}

Taking into account the asymptotic expansion for the Airy function [16],

$$
\operatorname{Ai}(-\mathrm{z}) \sim \frac{1}{\sqrt{\pi} \mathrm{z}^{1 / 4}}\left(\cos \left(\zeta-\frac{\pi}{4}\right) \sum_{\mathrm{s}=0}^{\infty}(-)^{\mathrm{s}} \frac{\mathrm{u}_{2 \mathrm{~s}}}{\zeta^{2 \mathrm{~s}}}+\sin \left(\zeta-\frac{\pi}{4}\right) \sum_{\mathrm{s}=0}^{\infty}(-)^{\mathrm{s}} \frac{\mathrm{u}_{2 \mathrm{~s}+1}}{\zeta^{2 \mathrm{~s}+1}}\right),
$$

with $\zeta=\frac{2}{3} z^{3 / 2}$ and $\mid$ phz $\mid<\frac{2}{3} \pi$, we have that

$$
\exists M, x_{0}<0 \quad \text { suchthat }|\operatorname{Ai}(\mathrm{x})-\overline{\operatorname{Ai}}(\mathrm{x})|<\frac{\mathrm{M}}{\mathrm{x}_{0}}, \mathrm{x}<\mathrm{x}_{0},
$$

where

$$
\overline{\operatorname{Ai}}(x)=\frac{(-x)^{-1 / 4}}{\sqrt{\pi}} \sin \left(\frac{2}{3}(-x)^{3 / 2}+\frac{\pi}{4}\right) .
$$

So we can write

$$
\begin{aligned}
S_{n}^{\mathrm{Ai}} & =-\int_{x_{0}}^{\infty} \mathrm{Ai}^{2}(x) \ln \mathrm{Ai}^{2}(x) d x-\int_{\beta_{n}}^{x_{0}} \mathrm{Ai}^{2}(x) \ln \mathrm{Ai}^{2}(x) d x \\
& \simeq-\int_{x_{0}}^{\infty} \mathrm{Ai}^{2}(x) \ln \mathrm{Ai}^{2}(x) d x-\int_{\beta_{n}}^{x_{0}} \overline{\mathrm{Ai}}^{2}(x) \ln \overline{\mathrm{Ai}}^{2}(x) d x \\
& =-\int_{x_{0}}^{\infty} \mathrm{Ai}^{2}(x) \ln \mathrm{Ai}^{2}(x) d x+\int_{x_{0}}^{0} \overline{\mathrm{Ai}}^{2}(x) \ln \overline{\mathrm{Ai}}^{2}(x) d x \\
& -\int_{\beta_{n}}^{0} \overline{\mathrm{Ai}}^{2}(x) \ln \overline{\mathrm{Ai}}^{2}(x) d x .
\end{aligned}
$$

Then,

$$
S_{n}^{\mathrm{Ai}} \simeq-\int_{\beta_{n}}^{0} \overline{\mathrm{Ai}}^{2}(x) \ln \overline{\mathrm{Ai}}^{2}(x) d x+\kappa,
$$

where

$$
\kappa=\lim _{x_{0} \rightarrow-\infty}\left(-\int_{x_{0}}^{\infty} \operatorname{Ai}^{2}(x) \ln \operatorname{Ai}^{2}(x) d x+\int_{x_{0}}^{0} \overline{\mathrm{Ai}}^{2}(x) \ln \overline{\mathrm{Ai}}^{2}(x) d x\right) .
$$


Using the $L^{q}$-norm method (see e.g. $[17,18]$ ) we can write

$$
-\int_{\beta_{n}}^{0} \overline{\mathrm{Ai}}^{2}(x) \ln \overline{\mathrm{Ai}}^{2}(x) d x=-\left[\frac{d}{d q} \int_{\beta_{n}}^{0}\left(\overline{\mathrm{Ai}}^{2}(x)\right)^{q} d x\right]_{q=1} .
$$

Considering the asymptotics of the zeros $\beta_{n}[16]$,

$$
\begin{array}{ll}
a_{s}=-T\left(\frac{3}{8} \pi(4 s-1)\right), & T(t) \sim t^{2 / 3}\left(1+\frac{5}{48} t^{-2}-\cdots\right), \\
a_{s}^{\prime}=-U\left(\frac{3}{8} \pi(4 s-3)\right), \quad U(t) \sim t^{2 / 3}\left(1-\frac{7}{48} t^{-2}+\cdots\right),
\end{array}
$$

as well as the change of variable, $x=\beta_{n}(y / \pi)^{2 / 3}$, and applying the Lemma 2.1 of Aptekarev et al. [17], which states that

$$
\int_{0}^{\pi} g(n \theta+\gamma(\theta)) f(\theta) \rightarrow{ }_{(n \rightarrow \infty)} \frac{1}{\pi} \int_{0}^{\pi} g(\theta) d \theta \int_{0}^{\pi} f(\theta) d \theta
$$

where $g \in C(\mathbf{R}), g(\theta+\pi)=g(\theta), f \in L^{1}([0, \pi])$, and $\gamma(\theta)$ measurable almost everywhere and finite in $[0, \pi]$; we have from Eq. (7),

$$
\begin{aligned}
& \int_{\beta_{n}}^{0}\left(\overline{\operatorname{Ai}}^{2}(x)\right)^{q} d x \\
& \frac{1}{\pi^{q}} \frac{2}{3}\left[\left(\frac{3}{4} n\right)^{\frac{2-q}{3}}+O\left(n^{-\frac{1+q}{3}}\right)\right]\left[\int_{0}^{\pi}\left[\sin ^{2}\left(\frac{1}{2} n y+\frac{1}{4}(y+\pi)\right)\right]^{q} y^{-\frac{q+1}{3}} d y+O\left(n^{-1}\right)\right] \\
& \rightarrow(n \rightarrow \infty) \frac{1}{\pi^{q}} \frac{2}{3}\left(\frac{3}{4} n\right)^{\frac{2-q}{3}} \\
& \times\left[\frac{1}{\pi} \int_{0}^{\pi}\left[\sin ^{2}(y)\right]^{q} d y \int_{0}^{\pi} y^{-\frac{q+1}{3}} d y+\tau(q) n^{\sigma(q)}\right]
\end{aligned}
$$

It can be seen that the limit set up by this lemma has an error term that goes as $\tau(q) n^{\sigma(q)}$, where we make the numerically-based hypothesis $\sigma(q)=\frac{q-2}{3}$ (see Table 3 in Section 4), so that the multiplicative factor $n^{\frac{2-q}{3}}$ cancels the $n$-dependence in the error term but not the $q$-dependence.

Thus, according to Eqs. (7) and (8), differentiating with respect to $q$ and taking $q=1$, we obtain the additional constant $\kappa^{\prime}$ in the limit,

$$
-\int_{\beta_{n}}^{0} \overline{\mathrm{Ai}}^{2}(x) \ln \overline{\mathrm{Ai}}^{2}(x) d x=C\left[\frac{1}{3} n^{\frac{1}{3}} \ln n+D n^{\frac{1}{3}}\right]+\kappa^{\prime}+O\left(n^{-\frac{2}{3}} \ln n\right),
$$

where $C=\frac{1}{\pi}\left(\frac{3}{4} \pi\right)^{\frac{1}{3}}, D=\frac{1}{3} \ln \left(48 \pi^{4}\right)-2$, and

$$
\kappa^{\prime}=-\frac{1}{3 \pi}\left(\frac{2}{9}\right)^{\frac{1}{3}}\left[\left(\log \frac{4}{3}-3 \log \pi\right) \tau(1)+3 \tau^{\prime}(1)\right] .
$$

Eqs. (5) and (9) provide the following asymptotical behavior of the entropy of the Airy function,

$$
S_{n}^{\mathrm{Ai}}=C\left[\frac{1}{3} n^{\frac{1}{3}} \ln n+D n^{\frac{1}{3}}\right]+\kappa+\kappa^{\prime}+O\left(n^{-\frac{2}{3}} \ln n\right) .
$$


For completeness, let us write down that the Boltzmann-Shannon information entropy (4) of the linear potential wavefunctions for the highly excited particle state is consequently given by

$$
S\left[\Psi_{n}\right]=\frac{2}{3} \ln n+\left(\ln \left(\frac{2(6 \pi)^{2 / 3}}{\alpha}\right)-2\right)+\frac{\kappa+\kappa^{\prime}}{C} n^{-\frac{1}{3}}+O\left(n^{-1} \ln n\right) .
$$

As already pointed out, the leading term was previously computed within the framework of WKB approximation by Sánchez-Ruiz [13]. Moreover, the same author on the basis of some numerical experiments, also conjectured the following term in the form $K n^{-1 / 3}$ with $K=0.709$ by taking into account only the odd-parity eigenfunctions; one can easily find (see e.g. Section 1 ) with the same method that $K=0.8933$ when both odd-parity and even-parity levels are considered.

The value of our work is to have determined the first two asymptotical terms of the information entropy by means of only the specific properties of the Airy function. However, we have not been able to explicitly compute the coefficient of the $n^{-1 / 3}$-term, i.e. the constant parameters $\kappa$ and $\kappa^{\prime}$. This will be done numerically in the next section.

\section{Numerical analysis.}

Now we turn to the numerical problem of computing the constants $\kappa$ and $\kappa^{\prime}$. We achieve this by doing various computations in order to obtain their values from different approaches.

First, we try to compute $\kappa$ from Eq. (5),

$$
\kappa=\lim _{n \rightarrow \infty}\left(S_{n}^{\mathrm{Ai}}+\int_{0}^{\beta_{n}} \overline{\mathrm{Ai}}^{2}(x) \ln \overline{\mathrm{Ai}}^{2}(x) d x\right) .
$$

The numerical study of this expression is shown in Table 1, where one can realize that $\kappa \simeq 0.2265$.

An alternative way to compute this constant is directly from its definition (6)

$$
\kappa=\lim _{x_{0} \rightarrow-\infty}\left(-\int_{x_{0}}^{+\infty} \mathrm{Ai}^{2}(x) \log \mathrm{Ai}^{2}(x) d x+\int_{x_{0}}^{0} \overline{\mathrm{Ai}}^{2}(x) \log \overline{\mathrm{Ai}}^{2}(x) d x\right),
$$

and the resulting values are given in Table 2 , where it is apparent that $\kappa \simeq 0.2265$.

At this it is pertinent to perform a numerical test of the hypothesis $\sigma(q)=\frac{q-2}{3}$. This is shown in Table 3, where we realize the high quality of the hypothesis. To obtain the $\sigma(q)$ values, a linear dependence on $q$ of the quantity $\sigma$ was assumed, and the numerical analysis of Eq. 8 was consequently performed.

The constant $\kappa^{\prime}$ can be obtained from the expression (9),

$$
\kappa^{\prime}=\lim _{n \rightarrow \infty}\left(-\int_{\beta_{n}}^{0} \overline{\operatorname{Ai}}^{2}(x) \ln \overline{\operatorname{Ai}}^{2}(x) d x-C\left[\frac{1}{3} n^{\frac{1}{3}} \ln n+D n^{\frac{1}{3}}\right]\right),
$$

whose numerical analysis is carried out in Table 4 , which shows that $\kappa^{\prime} \simeq 0.152$.

With these computations we find that $\kappa+\kappa^{\prime} \simeq 0.378$. Finally, two more tests for the $\kappa+\kappa^{\prime}$ value are possible. Firstly, by use of Eq. (10), one has

$$
\kappa+\kappa^{\prime}=\lim _{n \rightarrow \infty}\left(S_{n}^{\mathrm{Ai}}-C\left[\frac{1}{3} n^{\frac{1}{3}} \ln n+D n^{\frac{1}{3}}\right]\right) .
$$


The numerical study of this equation is done in Table 5, which also illustrates that $\kappa+\kappa^{\prime} \simeq 0.3785$.

The second and final test uses Eq. (11), which allows us to write down

$$
\kappa+\kappa^{\prime}=\lim _{n \rightarrow \infty}\left(\left[S\left[\Psi_{n}\right]-\frac{2}{3} \ln n+\left(\ln \left(\frac{2(6 \pi)^{2 / 3}}{\alpha}\right)-2\right)\right] C n^{\frac{1}{3}}\right) .
$$

The numerical study of this equation (see Table 6) leads again to the value $\kappa+\kappa^{\prime} \simeq 0.378$.

So, the coefficient for $n^{-1 / 3}$ term is $K=0.8936$, quite close to the $K=0.8933$ value given in [13].

\section{Summary and open problems.}

We have determined the asymptotics of the information entropy of the Airy function $\operatorname{Ai}(x)$, whose usefulness for the computation of the Boltzmann-Shannon entropy of the highly-energetic (Rydberg) quantum-mechanical states of the linear potential with vanishing angular momenta is also shown. In doing so the only existing related results [13], which are based in the WKB approximation, are rigorously corroborated.

Let us point out, for completeness, that the physical entropies of the non-vanishing angular momentum Rydberg states of the linear potential [19-21], so much useful for the theory of quark confinement, require the previous knowledge of the information entropies of the first order derivatives of the Airy function $\operatorname{Ai}^{\prime}(x)$ as well as the combination of $\operatorname{Ai}(x)$ and $\operatorname{Ai}^{\prime}(x)$. These two problems have not yet been solved.

\section{Acknowledgments}

We are grateful to J. Sánchez-Ruiz for discussions and sugestions. This work has been partially supported by the MCYT Project No. BFM2001-3878-C02-01, as well as by the NATO collaborative grant PST.CLG.929738. We belong to ehe J.A. Group FQM-207.

\section{References}

[1] C.E. Shannon, A mathematical theory of communication, Bell Syst. Tech. J. 27 (1948) 379-423, 623-656; Reprinted in: The Mathematical Theory of Communication, Eds. C.E. Shannon and W. Weaver, University of Illinois Press, Urbana, 1949

[2] M. Ohya and D.Petz, Quantum Information Entropy and Its Use, Springer Verlag, N.Y., 1993

[3] J.S. Dehesa, A. Martínez-Finkelshtein and J. Sánchez-Ruiz, Quantum information entropies and orthogonal polynomials, J. Comput. Appl. Math. 133 (2001) 23

[4] V. Buyarov, J.S. Dehesa, A. Martínez-Finkelshtein and J. Sánchez-Lara, Computation of the entropy of the polynomials orthogonal on an interval, SIAM J. Scientific Computing (2004). In press.

[5] J.S. Dehesa, A. Martínez-Finkelshtein and V.N. Sorokin, Short-wave asymptotics of the information entropy of a circular membrane, Int. J. Bifurcation and Chaos 12 (2002) 2387

[6] J.S. Dehesa, A. Martínez-Finkelshtein and V.N. Sorokin, Asymptotics of information entropies of some Toda-like potentials, J. Math. Phys. 44 (2003) 1 
[7] J.S. Dehesa, A. Martínez-Finkelshtein and V.N. Sorokin, Quantum-information entropies for highly-excited states of single-particle systems with power-type potentials, Physical Rev. A 66 (2002) 062109

[8] C.L. Critchfield, Scalar potentials in the Dirac equation, J. Math. Phys. 17 (1976) 261

[9] B.J. Harrington, S.Y. Park and A. Yildiz, Spectrum of heavy mesons in $e^{+} e^{-}$annihilation, and Orbital excitations in charmonium, Phys. Rev. Lett. 34 (1975) 168 and 706

[10] E.Eichten, K. Gottfried, T. Kinoshita, J. Kogut, K.D. Lane and T.M. Yam, Spectrum of charmed quark-antiquark bound states, Phys. Rev. Lett. 34 (1975) 369; Interplay of confinement and decay in the spectrum of charmonium, ibid 36 (1976) 500

[11] J.J. Aubert et al., Experimental observation of a heavy particle J, Phys. Rev. Lett. 33 (1974) 1404

[12] J.E. Augustin et al., Discovery of a narrow resonance in $e^{+} e^{-}$annihilation, Phys. Rev. Lett. 33 (1974) 1406

[13] J. Sánchez-Ruiz, Asymptotic formula for the quantum entropy of position in energy eigenstates, Phys. Lett. A 226 (1997) 7

[14] R.W. Robinett, Quantum and classical probability distributions for position and momentum, Am. J. Phys. 63 (1995) 823

[15] O. Valle and M. Soares, Les Fonctions d'Airy pour la Physique, Diderot, 1998

[16] F.W. Olver, Airy and Related Functions, in Digital Library of Mathematical Functions, WWW address: http://dlmf.nist.gov/

[17] A.I. Aptekarev, V. Buyarov and J.S. Dehesa, Asymptotic behaviour of $L_{p}$-norms and entropy for general orthogonal polynomials, RAS Sbornik Math 82 (1995) 373

[18] R.J. Yáñez, Information entropy, orthogonal polynomials and multielectronic systems, $\mathrm{PhD}$ Thesis (in Spanish), Universidad de Granada, 1995.

[19] A.F. Antippa and A.J. Phares, The linear potential: a solution in terms of combinatorics functions, J. Math. Phys. 19 (1978) 308

[20] A.J. Phares, The energy eigenvalue equation for the linear potential, J. Math. Phys. 19 (1978) 2239

[21] P.A. Maurone and A.J. Phares, The linear potential wavefunctions, J. Math. Phys. 21 (1980) 830 
Table 1. Numerical study of Eq. (12).

\begin{tabular}{rlll}
\hline $\mathrm{n}$ & $S_{n}^{\mathrm{Ai}}$ & $-\int_{0}^{\beta_{n}} \overline{\mathrm{Ai}}^{2}(x) \ln \overline{\mathrm{Ai}}^{2}(x) d x$ & $\kappa$ \\
\hline 1 & 0.8491255877133055 & 0.6180634559349288 & 0.2310621317783767 \\
2 & 1.016910182186539 & 0.7907991368271019 & 0.2261110453594368 \\
5 & 1.415460253243194 & 1.188199380524827 & 0.2272608727183667 \\
10 & 1.862037026152716 & 1.635594254035353 & 0.2264427721173636 \\
20 & 2.491976409618374 & 2.265497102260039 & 0.2264793073583347 \\
50 & 3.703731382087362 & 3.477237747557407 & 0.2264936345299549 \\
100 & 5.012945106156195 & 4.786446149442159 & 0.2264989567140363 \\
200 & 6.783473208118115 & 6.556977015888961 & 0.2264961922291544 \\
500 & 10.09256633814676 & 9.866065338606688 & 0.2265009995400753 \\
1000 & 13.59354960534267 & 13.36705097438357 & 0.2264986309591066 \\
10000 & 35.84815107795398 & 35.62158882912692 & 0.2265622488270580 \\
100000 & 91.88389679603820 & 91.65736391453106 & 0.2265328815071399 \\
\hline
\end{tabular}


Table 2. Numerical study of Eq. (13).

\begin{tabular}{rlll}
\hline$x_{0}$ & $-\int_{x_{0}}^{+\infty} \mathrm{Ai}^{2}(x) \log \mathrm{Ai}^{2}(x) d x$ & $\int_{x_{0}}^{0} \overline{\mathrm{Ai}}^{2}(x) \log \overline{\mathrm{Ai}}^{2}(x) d x$ & $\kappa$ \\
\hline-1 & 0.5222161751978991 & 0.2961890404907877 & 0.2260271347071114 \\
-10 & 2.077338083778954 & 1.850652129535547 & 0.2266859542434068 \\
-100 & 9.397399253933362 & 9.170902650261821 & 0.2264966036715403 \\
-200 & 14.69392229097821 & 14.46742682065904 & 0.2264954703191755 \\
-500 & 26.27530667657610 & 26.04880146831822 & 0.2265052082578833 \\
-1000 & 40.49014289623582 & 40.26363079325318 & 0.2265121029826460 \\
\hline
\end{tabular}


Table 3. Numerical analysis of the hypothesis $\sigma(q)=\frac{q-2}{3}$

\begin{tabular}{lll}
\hline$q$ & $\sigma(q)$ & $(q-2) / 3$ \\
\hline 0.5 & -0.4990839447495011 & -0.5 \\
0.9 & -0.3659978669311457 & -0.366666 \\
0.95 & -0.3493617633586690 & -0.35 \\
0.99 & -0.3360528305771765 & -0.336666 \\
0.995 & -0.3343892108870942 & -0.335 \\
0.999 & -0.3330583146412395 & -0.333666 \\
1.0 & -0.3327255905122667 & -0.333333 \\
1.001 & -0.3323928663558781 & -0.333 \\
1.005 & -0.3310619694535155 & -0.331666 \\
1.01 & -0.3293983477085469 & -0.33 \\
1.05 & -0.3160893490880808 & -0.316666 \\
1.1 & -0.2994530387476477 & -0.3 \\
1.5 & -0.1663586884534043 & -0.166666 \\
\hline
\end{tabular}


Table 4. Numerical study of Eq. (14).

\begin{tabular}{rll}
\hline$n$ & $-\int_{\beta_{n}}^{0} \overline{\mathrm{Ai}}^{2}(x) \ln \overline{\mathrm{Ai}}^{2}(x) d x$ & $\kappa^{\prime}$ \\
\hline 1 & 0.6180634559349288 & 0.2721346681876156 \\
2 & 0.7907991368271019 & 0.2316547650211527 \\
5 & 1.188199380524827 & 0.2081046520126155 \\
10 & 1.635594254035353 & 0.1899098287874792 \\
20 & 2.265497102260039 & 0.1784040275788774 \\
50 & 3.477237747557407 & 0.1680146216219147 \\
100 & 4.786446149442159 & 0.1628399946896453 \\
200 & 6.556977015888961 & 0.1592838456040866 \\
500 & 9.866065338606688 & 0.1562518872093719 \\
1000 & 13.36705097438357 & 0.1548086738545216 \\
10000 & 35.62158882912692 & 0.1526412373166366 \\
100000 & 91.65736391453106 & 0.1520980222152417 \\
1000000 & 229.8043910824926 & 0.1524238466430177 \\
\hline
\end{tabular}


Table 5. Numerical study of Eq. (15).

\begin{tabular}{rll}
\hline$n$ & $S_{n}^{\mathrm{Ai}}$ & $\kappa+\kappa^{\prime}$ \\
\hline 1 & 0.8491255877133055 & 0.5031967999659922 \\
2 & 1.016910182186540 & 0.4577658103805904 \\
5 & 1.415460253243193 & 0.4353655247309812 \\
10 & 1.862037026141317 & 0.4163526008934437 \\
20 & 2.491976409144777 & 0.4048833344636149 \\
50 & 3.703731017196829 & 0.3945078912613367 \\
100 & 5.012945102393711 & 0.3893389476411979 \\
200 & 6.783473041810359 & 0.3857798715254850 \\
500 & 10.09256605054762 & 0.3827525991503027 \\
1000 & 13.59354798908833 & 0.3813056885592871 \\
10000 & 35.84808970400448 & 0.3791421121941951 \\
100000 & 91.88385127364121 & 0.3785853813253937 \\
1000000 & 230.0304243971540 & 0.3784571613044250 \\
10000000 & 565.1890275970421 & 0.3785182856238407 \\
\hline
\end{tabular}


Table 6. Numerical study of Eq. (16).

\begin{tabular}{rll}
\hline$n$ & $S\left(\Psi_{n}\right)$ & $\kappa+\kappa^{\prime}$ \\
\hline 1 & 1.710183690538731 & 0.4487154478408955 \\
2 & 1.914670354838653 & 0.4278693661686791 \\
5 & 2.294432719271654 & 0.4133267764578933 \\
10 & 2.625943420152888 & 0.4015923330453082 \\
20 & 2.990723472091418 & 0.3940847941662819 \\
50 & 3.507127798799131 & 0.3874632791011496 \\
100 & 3.916389626903620 & 0.3842961155825298 \\
200 & 4.337317744851943 & 0.3822036132202163 \\
500 & 4.907064452326643 & 0.3805121161752789 \\
1000 & 5.345631824193879 & 0.3797471090953328 \\
10000 & 6.832532675670682 & 0.3786967516781765 \\
100000 & 8.345340678998884 & 0.3784637933050010 \\
1000000 & 9.870081112950714 & 0.3784129459145020 \\
\hline
\end{tabular}

